\title{
Generation of guided space-time wave packets using multilevel indirect photonic transitions in integrated photonics
}

\author{
Cheng Guo $\odot^{1, *}$ and Shanhui Fan ${ }^{2, \dagger}$ \\ ${ }^{1}$ Department of Applied Physics, Stanford University, Stanford, California 94305, USA \\ ${ }^{2}$ Ginzton Laboratory and Department of Electrical Engineering, Stanford University, Stanford, California 94305, USA
}

(Received 7 June 2021; accepted 9 August 2021; published 17 August 2021)

\begin{abstract}
We propose an integrated photonic approach to generating guided space-time wave packets in a conventional multimode waveguide using multilevel interband photonic transitions. It can generate broadband propagationinvariant space-time wave packets with arbitrary group velocity and tailored field profiles. Our work reveals an important connection between the study of time-varying media and space-time optics.
\end{abstract}

DOI: 10.1103/PhysRevResearch.3.033161

\section{INTRODUCTION}

There have been emerging interests in exploring nontrivial optical phenomena in time-varying media [1-10]. In particular, photonic transitions induced by dynamic modulation [11-15] have enabled unconventional opportunities in integrated photonics [16,17], such as optical isolation [13,18-22], circulation [23], effective magnetic field, [24,25], photonic Aharonov-Bohm effect [26-28], and slow light [14]. However, the majority of the existing works have focused only on transitions between a few photonic levels.

In this work, we consider photonic transitions in a waveguide consisting of a large number of modes. We show that for this system the photonic transitions can result in the creation of space-time wave packets in the waveguide. Space-time wave packets [29-31] are a special class of wave packets that are propagation invariant [32-39], i.e., these wave packets do not suffer from dispersion or diffraction under propagation. Such wave packets were first studied in free space [29,40,41] or bulk media $[42,43]$. Creating space-time wave packets in a waveguide [44-46] may lead to new opportunities in multimode integrated photonics. For example, these wave packets can propagate at arbitrarily low group velocity but still possess broad spectral bandwidth.

Generating a space-time wave packet in general is nontrivial due to the specific spatiotemporal correlation [30,47]. To generate such a wave packet in a waveguide presents additional difficulties. Previous attempts either first synthesized a wave packet in free space and then coupled it into the waveguide [44], or relied upon strong nonlinearity [46]. In contrast, in our approach [Fig. 1(a)], we first excite an eigenmode of a multimode waveguide [Fig. 1(b)], and then transmit the light

\footnotetext{
*guocheng@stanford.edu

†shanhui@stanford.edu
}

Published by the American Physical Society under the terms of the Creative Commons Attribution 4.0 International license. Further distribution of this work must maintain attribution to the author(s) and the published article's title, journal citation, and DOI. through a traveling wave modulator. We show that, with a suitable design, such a modulator can convert such an excitation into a space-time wave packet [Figs. 1(c) and 1(d)]. The group velocity of the wave packet is controlled by the phase velocity of the traveling wave modulator and can be arbitrarily tuned. Our work highlights an important connection between the study of time-varying media and space-time optics.

The rest of this paper is organized as follows. In Sec. II we provide the theory. In Sec. III we provide numerical demonstrations. We discuss and conclude in Sec. IV. The Appendix provides a supplemental discussion.

\section{THEORY}

To start, we briefly review propagation-invariant spacetime wave packets (also termed "discretized conical waves" [46]) in multimode waveguides. For concreteness, we consider a planar multimode waveguide with a dielectric permittivity distribution $\varepsilon_{s}(x)$. We focus on the TE modes propagating in the $z$ direction with an electric field $E(x, z, t)$ along the $y$ direction (Fig. 1). The $\nu$ th eigenmode has its electric field $E(x, z, t)=\phi_{\nu}(x ; \omega) \exp \left[-i \omega t+i \beta_{\nu}(\omega) z\right]$, where $\beta_{v}(\omega)$ is the propagation constant, and the modal profile $\phi_{v}(x ; \omega)$ satisfies

$$
\left[\frac{d^{2}}{d x^{2}}+\frac{\omega^{2}}{c^{2}} \varepsilon_{s}(x)\right] \phi_{\nu}(x ; \omega)=\beta_{v}^{2}(\omega) \phi_{\nu}(x ; \omega) .
$$

For subsequent use, the modal profiles are chosen so that a mode with unit amplitude carries unit photon number flux.

A guided space-time wave packet is a linear superposition of selected eigenmodes over multiple bands:

$$
\begin{aligned}
E(x, z, t) & =\sum_{\nu=0}^{n-1} a_{\nu} \phi_{\nu}\left(x ; \omega_{\nu}\right) e^{-i \omega_{\nu} t+i \beta_{\nu}\left(\omega_{\nu}\right) z} \\
& \equiv e^{-i \omega_{0} t+i \beta_{0}\left(\omega_{0}\right) z} A(x, z, t),
\end{aligned}
$$

where

$$
A(x, z, t)=\sum_{\nu=0}^{n-1} a_{\nu} \phi_{\nu}\left(x ; \omega_{\nu}\right) e^{-i\left(\omega_{\nu}-\omega_{0}\right) t+i\left[\beta_{\nu}\left(\omega_{\nu}\right)-\beta_{0}\left(\omega_{0}\right)\right] z} .
$$


(a)

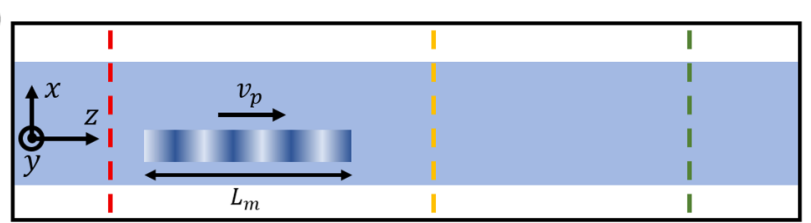

(b)

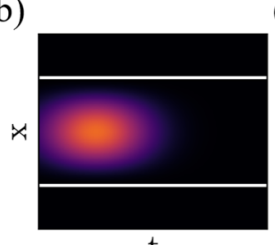

t

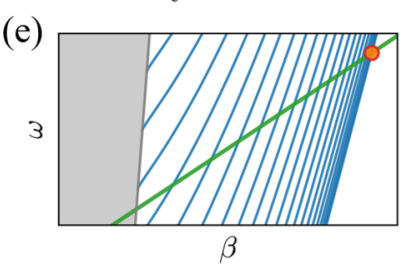

(c)

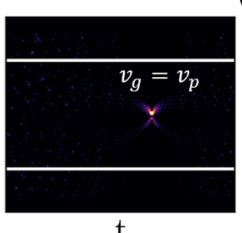

(d)

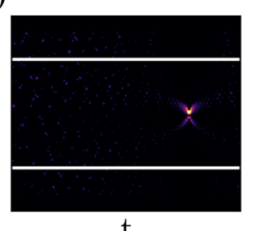

$\mathrm{t}$

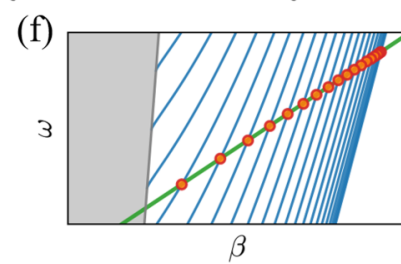

FIG. 1. Scheme. (a) A multimode waveguide with a region of length $L_{m}$ that is modulated by a traveling wave with phase velocity $v_{p}$. The light blue region indicates the guiding region of the waveguide. Light propagates in the $z$-direction. (b) The incident light [at the red line in (a)] is approximately an eigenstate of the static waveguide. (c), (d) The transmitted light [at the yellow and green line in (a)] is a propagation-invariant space-time wave packet with a group velocity $v_{g}=v_{p}$. (e), (f) The spatiotemporal spectrum of the incident (e) and transmitted light (f) on the dispersion relation of the waveguide (blue curves). $\omega$ and $\beta$ are the frequencies and the wave vector, respectively. The selected eigenmodes (orange dots) lie on a straight line specified by the traveling wave modulation.

In Eqs. (2) and (3), $\omega_{0}$ and $\beta_{0}\left(\omega_{0}\right)$ are the carrier frequency and wave vector, respectively; $A(x, z, t)$ is the complex envelope; $a_{v}$ is the complex mode amplitude, and $\left|a_{v}\right|^{2}$ is the photon number flux carried by the $\nu$ th mode. Importantly, the selected eigenmodes exhibit a specific spatiotemporal spectral correlation [Figs. 1(e) and 1(f)]:

$$
\omega_{v}=v_{g}\left[\beta_{\nu}\left(\omega_{\nu}\right)-\beta_{0}\left(\omega_{0}\right)\right]+\omega_{0}=v_{g} \beta_{v}\left(\omega_{v}\right)+\omega_{b},
$$

where $\omega_{b}=\omega_{0}-v_{g} \beta_{0}\left(\omega_{0}\right)$. Therefore,

$$
\begin{aligned}
A(x, z, t) & =\sum_{\nu=0}^{n-1} a_{\nu} \phi_{\nu}\left(x ; \omega_{\nu}\right) e^{-i\left(\omega_{\nu}-\omega_{0}\right)\left(t-z / v_{g}\right)} \\
& =A\left(x, 0, t-z / v_{g}\right)
\end{aligned}
$$

thus the envelope propagates without distortion at a group velocity $v_{g}$.

Although the existence of such space-time wave packets in a waveguide has recently been noted [46], it remains highly nontrivial to generate such guided space-time wave packets. The challenges are twofold. First, the spatiotemporal correlation of Eq. (4) must be precisely achieved to ensure the propagation invariance and the desired group velocity. Second, the distribution of complex mode amplitudes $\left\{a_{v}\right\}$ must be precisely engineered to obtain a specific spatiotemporal field profile. (This point is further discussed in the Appendix.)

In this work, we show that both of these challenges can be effectively overcome with the use of multilevel indirect photonic transitions in a waveguide. Specifically, we apply a traveling wave modulation to a section of the waveguide:

$$
\varepsilon(x, z, t)=\varepsilon_{s}(x)+\delta \varepsilon(x, z, t),
$$

where $\varepsilon_{s}(x)$ describes the waveguide in the absence of modulation, and

$$
\delta \varepsilon(x, z, t)=\Delta \varepsilon(x) g\left(z-v_{p} t\right),
$$

where $\Delta \varepsilon(x)$ is the transverse modulation profile, and the arbitrary function $g(\cdot)$ describes the longitudinal modulation profile, which has a traveling wave profile.

We consider an initial state that is an eigenmode of the static waveguide with frequency and wave vector $\left(\omega_{i}, \beta_{i}\left(\omega_{i}\right)\right)$. The traveling wave modulation induces indirect photonic transitions [13] among $n$ eigenmodes in different bands with frequencies and wave vectors $\left(\omega_{\nu}, \beta_{\nu}\left(\omega_{\nu}\right)\right), v=0,1, \ldots, n-$ 1. Importantly, the traveling wave profile of Eq. (7) ensures that the phase-matching condition $\omega_{v}-\omega_{i}=v_{p}\left[\beta_{v}\left(\omega_{v}\right)-\right.$ $\left.\beta_{i}\left(\omega_{i}\right)\right]$ is satisfied in the transition process, which guarantees the crucial spatiotemporal correlation [Eq. (4)].

We next consider the challenge of creating a specific spatiotemporal field profile for the wave packet. Below we show that under a very general set of assumptions, by controlling only the length of the modulation region, any specific target wave packet can be achieved, using practically any modulation profiles as described by the functions $\delta \epsilon$ and $g$ in Eq. (7). To show this, as the starting point, in the modulated region,

$$
E(x, z, t)=\sum_{\nu=0}^{n-1} a_{\nu}(z) \phi_{\nu}\left(x ; \omega_{\nu}\right) e^{-i \omega_{\nu} t+i \beta_{\nu}\left(\omega_{\nu}\right) z},
$$

where $a_{v}(z)$ is the $z$-dependent complex amplitude of the $v$ th eigenmode. Substituting Eq. (8) into Maxwell's equations and using the slowly varying envelope approximation, one derives the spatial coupled mode equations:

$$
\frac{d}{d z}|\phi(z)\rangle=i H|\phi(z)\rangle,
$$

where $|\phi(z)\rangle=\left(a_{0}(z), a_{1}(z), \ldots, a_{n-1}(z)\right)^{T}$. When the coupling between all these eigenmodes are phase-matched, $H$ is $z$-independent.

Assuming $H$ is Hermitian, which is true for lossless modulated structure, then

$$
H=\sum_{i=0}^{n-1} \lambda_{i}\left|e_{i}\right\rangle\left\langle e_{i}\right|,
$$

where $\lambda_{i}$ and $\left|e_{i}\right\rangle$ are the eigenvalues and eigenstates of $H$, respectively. The initial state

$$
|\phi(0)\rangle=\sum_{i=0}^{n-1} \phi_{i}\left|e_{i}\right\rangle
$$

evolves as

$$
|\phi(z)\rangle=\sum_{i=0}^{n-1} \phi_{i} e^{i \lambda_{i} z}\left|e_{i}\right\rangle .
$$


(a)
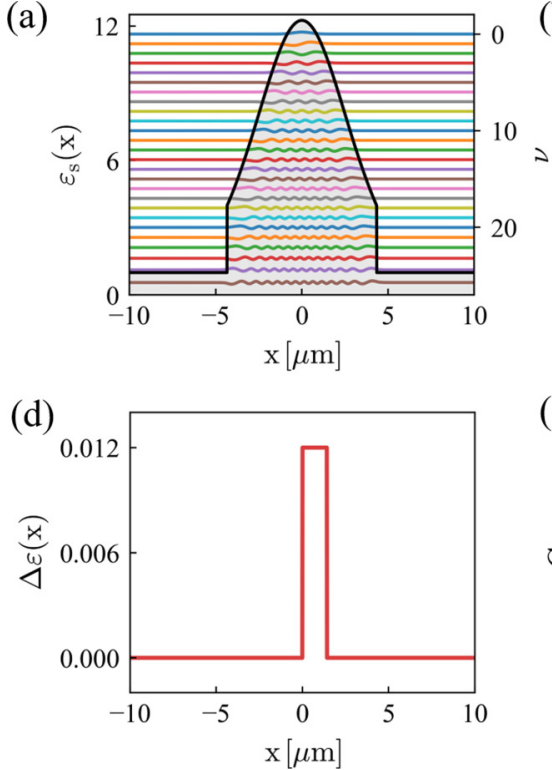

(b)

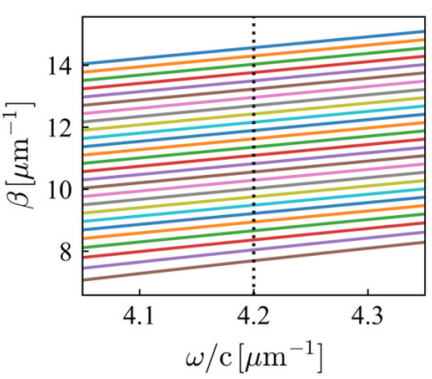

(e)

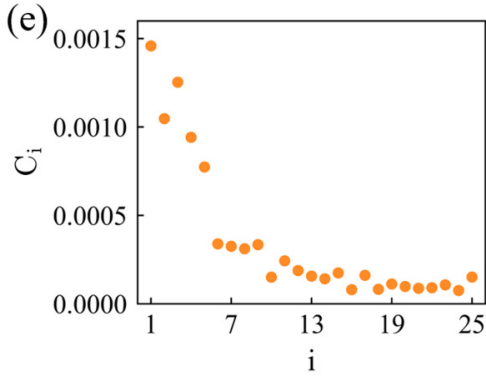

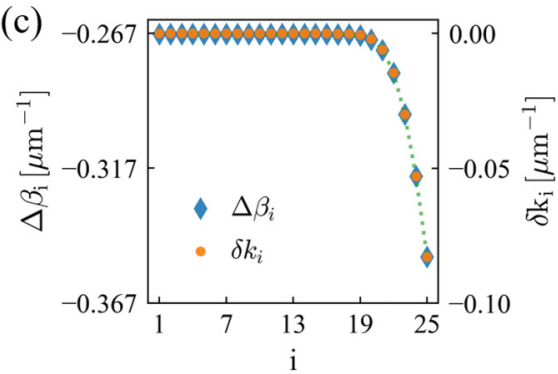

(f)

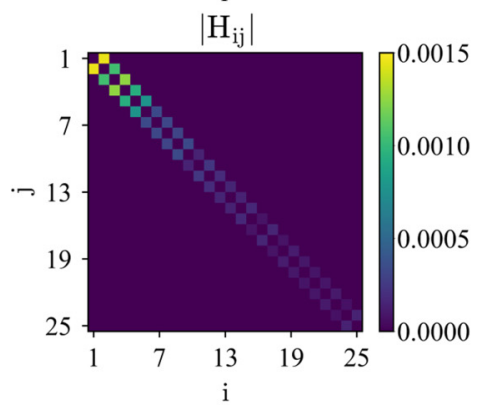

FIG. 2. GRIN waveguide and modulation. (a) Static dielectric constant profile $\varepsilon_{s}(x)$ (black) and eigenmodes $\phi_{\nu}\left(x ; \omega_{\nu}\right)$ (shifted vertically for clarity). (b) Propagation constant spectra $\beta_{v}(\omega)$. Dotted line: $\omega=v_{g} \beta+\omega_{b}$ where $\omega_{b} / c=4.2 \mu \mathrm{m}^{-1}, v_{g}=1 \times 10^{-5}$ c. Eigenmodes are selected at the intersections. (c) Wave vector differences $\Delta \beta_{i}$ and mismatches $\delta k_{i}$ between neighboring modes. $i$ labels the interval between the $(i-1)$-th and $i$ th mode. (d) Transverse modulation profile $\Delta \varepsilon(x)$. (e) Coupling strength $C_{i}$. (f) The magnitudes of the matrix elements of the Hamiltonian $\left|H_{i j}\right|$.

For a given target state

$$
\left|\psi_{t}\right\rangle=\sum_{i=0}^{n-1} \psi_{i}\left|e_{i}\right\rangle,
$$

we define a fidelity of $|\phi(z)\rangle$ as

$$
F(z) \equiv\left|\left\langle\psi_{t} \mid \phi(z)\right\rangle\right|=\left|\sum_{i=0}^{n-1} \psi_{i}^{*} \phi_{i} e^{i \lambda_{i} z}\right| .
$$

For a given initial state, the maximum fidelity that one can achieve is

$$
F \equiv \max _{z}\left|\left\langle\psi_{t} \mid \phi(z)\right\rangle\right|=\sum_{i=0}^{n-1}\left|\psi_{i}^{*} \phi_{i}\right|
$$

Generically, the different eigenvalues $\lambda_{i}$ of a Hamiltonian are incommensurate, thus the dynamics as described by Eq. (12) is quasiperiodic. According to Weyl's theorem [48] on the density of values of quasiperiodic functions [49], for any $\epsilon>0$, there exists a $z>0$ such that $|F(z)-F|<\epsilon$. Therefore, to approach a certain target state, we choose an initial state which can be readily generated with standard techniques and with a large $F$ value as defined by Eq. (15) with respect to the target state. We then choose the modulation length appropriately to maximize the fidelity of the final state. The discussion above guarantees that maximum fidelity can be reached. In practice, for a given maximum modulation strength, there are still substantial degrees of freedom in choosing the transverse modulation profile $\Delta \varepsilon(x)$. These degrees of freedom can be used to minimize the length of the modulation region.

\section{NUMERICAL DEMONSTRATION}

Now we provide a concrete numerical demonstration. To highlight the essential physics, we focus on monochromatic modulation. Accordingly, we choose a waveguide with an equally spaced eigenspectrum, which supports phase-matched multilevel photonic transitions under single-frequency modulation.

We consider transverse electric modes in the same setup as in Fig. 1(a) using a gradient index (GRIN) planar waveguide [50-53] with a refractive index profile [Fig. 2(a)]

$$
\varepsilon_{s}(x)= \begin{cases}n_{0}^{2} \operatorname{sech}^{2}(\alpha x), & |x| \leqslant x_{t} \\ n_{c}^{2}, & |x|>x_{t}\end{cases}
$$

where $n_{0}=3.5$ is the maximum refractive index, $n_{c}=1.0$ is the refractive index of cladding, $\alpha=0.2674 \mu \mathrm{m}^{-1}$ controls the width of refractive index variation, and $x_{t}$ is the truncation position of the refractive index variation. In a practical GRIN waveguide, $x_{t}$ is chosen such that $n_{0} \operatorname{sech}\left(\alpha x_{t}\right)>n_{c}$ [Fig. 2(a)].

For simplicity, we first consider the case without truncation, i.e., $x_{t} \rightarrow \infty$. Then Eq. (1) has analytical solutions of eigenmodes [54]

$$
\begin{aligned}
\phi_{\nu}(x ; \omega)= & c_{\nu} \operatorname{sech}^{S-\nu}(\alpha x) f_{\nu}(-v, 2 S-v+1, S \\
& \left.-v+1 ; \frac{1}{2}[1-\tanh (\alpha x)]\right)
\end{aligned}
$$

with eigenvalues

$$
\beta_{v}(\omega)=\alpha(S-v),
$$

where $v=0,1,2, \ldots, c_{v}$ is a normalization constant, $f_{v}$ is the hypergeometric function which is a polynomial of degree 
$\nu$, and

$$
S=1 / 2\left[\left(1+4 V^{2}\right)^{\frac{1}{2}}-1\right],
$$

where $V$ is the normalized width of the profile

$$
V(\omega)=\frac{n_{0} \omega}{\alpha c} .
$$

Equation (18) indicates an equally spaced propagation constant spectrum with a spacing:

$$
\Delta \beta_{v+1}(\omega) \equiv \beta_{v+1}(\omega)-\beta_{\nu}(\omega)=-\alpha .
$$

We note that the spacing is solely determined by $\alpha$ in Eq. (16) and independent of either $v$ or $k$.

Next, we introduce a finite truncation $x_{t}<\infty$. For simulation, we choose $x_{t}=4.346 \mu \mathrm{m}$. The truncation affects only the higher-order modes that have significant field intensity near the truncation. We solve Eq. (1) numerically using transfer matrix method [55]. Figure 2(a) plots the field distribution $\phi_{\nu}(x)$ of the eigenmodes at $\omega / c=4.2 \mu \mathrm{m}^{-1}(\nu=$ $0,1, \ldots, 25$ from top to bottom), which agree well with Eq. (17) when $v \leqslant 19$. Figure 2(b) plots the propagation constant spectrum $\beta_{v}(\omega)(v=0,1, \ldots, 25$ from top to bottom), which agree well with Eq. (18) for $v \leqslant 19$. Figure 2(c) plots the spacing of propagation constants $\Delta \beta_{v+1}(\omega) \equiv \beta_{v+1}(\omega)-$ $\beta_{\nu}(\omega)$ at $\omega / c=4.2 \mu \mathrm{m}^{-1}$. For $v \leqslant 19$, the spacing is constant $\Delta \beta_{v+1}=-\alpha=-0.2674 \mu \mathrm{m}^{-1}$ as expected from Eq. (21). For $v>19$, the propagation constants are no longer equally spaced.

We apply a monochromatic traveling wave modulation to a section of the waveguide,

$$
\delta \varepsilon(x, z, t)=\Delta \varepsilon(x) \cos (\Omega t-q z),
$$

where $\Omega$ and $q$ are the modulation frequency and wave vector, respectively. The phase velocity $v_{p}=\Omega / q$. For concreteness, we choose

$$
\Delta \varepsilon(x)=\Delta \varepsilon_{0} \times\left[\Theta(x)-\Theta\left(x-x_{1}\right)\right],
$$

where $\Theta(\cdot)$ is the Heaviside step function, $\Delta \varepsilon_{0}=1.2 \times 10^{-2}$, and $x_{1}=1.4212 \mu \mathrm{m}$ [Fig. 2(d)]. The relative modulation strength $\Delta \varepsilon_{0} / n_{0}^{2} \sim 10^{-3}$ is achievable for electro-optic modulators [56-58].

We choose the initial state to be an eigenmode of the static waveguide with frequency and wave vector $\left(\omega_{i}, \beta_{i}\left(\omega_{i}\right)\right)$, where $0 \leqslant i \leqslant 25$. (We note that selective excitation of a single eigenmode in a multimode waveguide has been previously demonstrated [59].) The modulation couples a set of eigenmodes with frequencies and wave vectors $\left(\omega_{\nu}, \beta_{\nu}\left(\omega_{\nu}\right)\right)$, where $0 \leqslant v \leqslant 25$ and $\omega_{v+1}-\omega_{\nu}=\Omega$. The dynamics is described by the coupled mode equations [Eq. (9)] with the Hamiltonian

$$
H=\left(\begin{array}{cccccc}
0 & C_{1} e^{-i \delta k_{1} z} & 0 & 0 & \ldots & 0 \\
C_{1} e^{i \delta k_{1} z} & 0 & C_{2} e^{-i \delta k_{2} z} & 0 & \cdots & 0 \\
0 & C_{2} e^{i \delta k_{1} z} & 0 & C_{3} e^{-i \delta k_{3} z} & \cdots & 0 \\
\vdots & & & \ddots & & \vdots \\
0 & \ldots & 0 & C_{n-2} e^{i \delta k_{n-2} z} & 0 & C_{n-1} e^{-i \delta k_{n-1} z} \\
0 & \cdots & 0 & 0 & C_{n-1} e^{i \delta k_{n-1} z} & 0
\end{array}\right)
$$

where the coupling strength

$$
C_{i}=\frac{\varepsilon_{0}}{8} \int_{-\infty}^{\infty} \Delta \varepsilon(x) \phi_{i}\left(x ; \omega_{i}\right) \phi_{i-1}\left(x ; \omega_{i-1}\right) d x,
$$

and the wave vector mismatch

$$
\delta k_{i}=\beta_{i}-\beta_{i-1}-q
$$

for $i=1,2, \ldots, n-1$.

We consider two cases of traveling wave modulation: (1) $\Omega^{(1)} / c=2.674 \times 10^{-6} \mu \mathrm{m}^{-1}, q^{(1)}=0.2674 \mu \mathrm{m}^{-1}$, thus $v_{p}^{(1)}=1.000 \times 10^{-5} \mathrm{c} . \quad(2) \quad \Omega^{(2)} / c=5.347 \times 10^{-6} \mu \mathrm{m}^{-1}$, $q^{(2)}=-0.2674 \mu \mathrm{m}^{-1}$, thus $v_{p}^{(2)}=-2.000 \times 10^{-5} \mathrm{c} . \Omega \sim$ $0.1-1 \mathrm{GHz}$ is typical for electro-optical modulators [56-58]. For each case, we calculate $C_{i}$ using Eq. (25) and $\delta k_{i}$ using Eq. (26), then construct $H$ using Eq. (24). Figures 2(c), 2(e), and 2(f) plot $C_{i}, \delta k_{i}$ and $H$ for case (1), respectively. The results are similar for case (2). $\left|\delta k_{i}\right|$ exhibits a behavior consistent with $\Delta \beta_{i}$. It is essentially zero for $i \leqslant 19$, but increases significantly when $i>19$. Due to the large phase mismatch, the transition amplitude is negligible for $v>19$ [13].

In the first example, we use the modulation of case (1) to generate a train of slow guided space-time wave packets with a group velocity $v_{g}^{(1)}=1 \times 10^{-5} \mathrm{c}$. The modulation induces

photonic transitions among a set of eigenmodes [green dots in Fig. 3(a)] that approximately satisfies the spatiotemporal correlation

$$
\omega_{v}^{(1)}-\omega_{0}^{(1)}=v_{p}^{(1)}\left(\beta_{v}^{(1)}-\beta_{0}^{(1)}\right),
$$

where $\omega_{0}^{(1)} / c=4.2+13.59 \times 10^{-5} \mu \mathrm{m}^{-1}, \beta_{0}^{(1)}=\beta_{0}\left(\omega_{0}\right)=$ $14.5674 \mu \mathrm{m}^{-1}$. Equation (27) is satisfied almost perfectly for $v \leqslant 19$.

To demonstrate the versatility of our approach, we choose an arbitrary target state with a mode amplitude distribution [Fig. 3(d)]:

$$
a_{\nu}=\left\{\begin{array}{ll}
a_{0} e^{-\gamma \nu}, & v=0,2, \ldots, 20 \\
i a_{0} e^{-\gamma \nu}, & v=1,3, \ldots, 19 \\
0, & v=21,22, \ldots, 25
\end{array},\right.
$$

where $a_{0}=0.4634$ and $\gamma=0.12$ such that $\sum_{\nu=0}^{25}\left|a_{\nu}\right|^{2}=$ 1. Figure 3(e) shows the intensity distribution $|E(x, t)|^{2}$ of the target state at $z=0 \mu \mathrm{m}$, which exhibits a train of light pulses with a glide symmetry of periodicity $d^{(1)}=2 \pi / q^{(1)}=$ $23.50 \mu \mathrm{m}$. Figure 3(f) shows $|E(x, t)|^{2}$ of the target state at $z=5 \mu \mathrm{m}$. Compared with Fig. 3(e), the whole intensity distribution is indeed delayed by $\tau^{(1)}=\Delta z / v_{g}^{(1)}=1.67 \mathrm{~ns}$. 
(a)

(d)
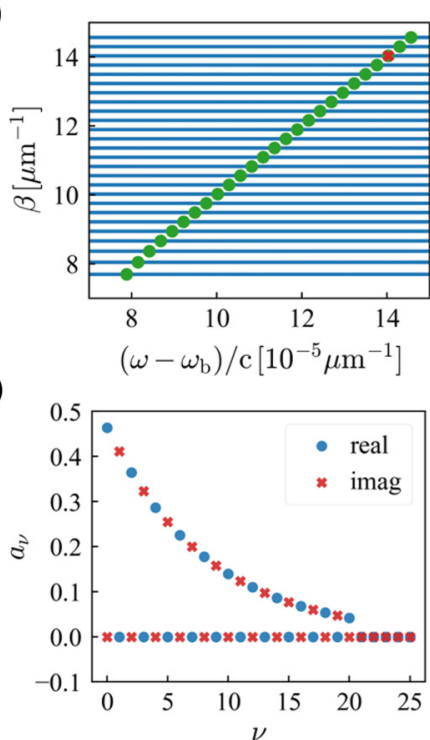

$(\mathrm{g})$

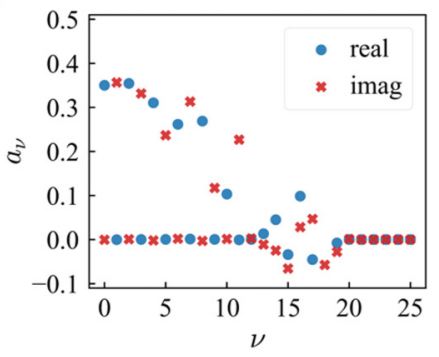

(b)

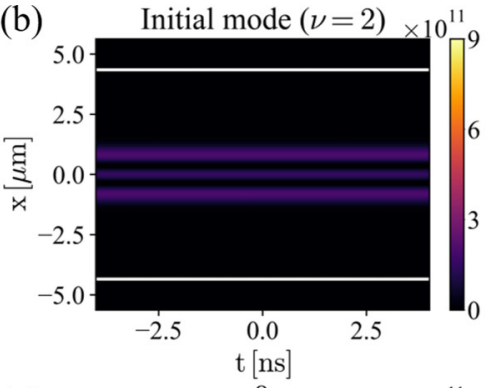

(e)

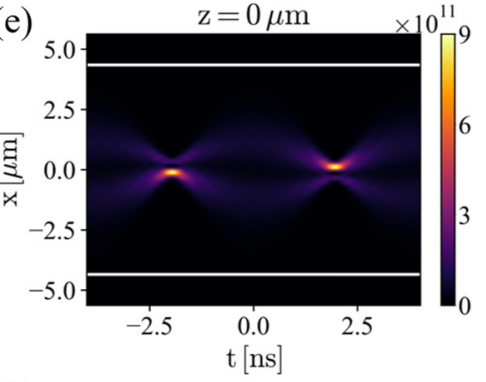

(h)

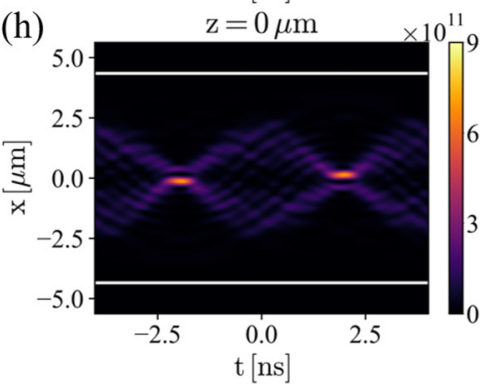

(c)

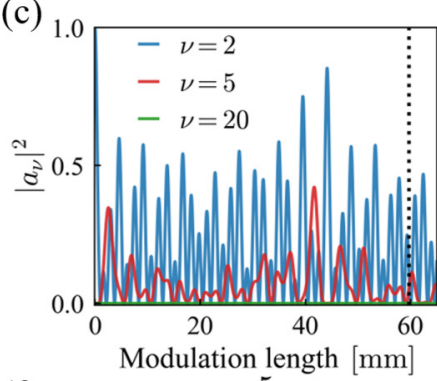

(f)

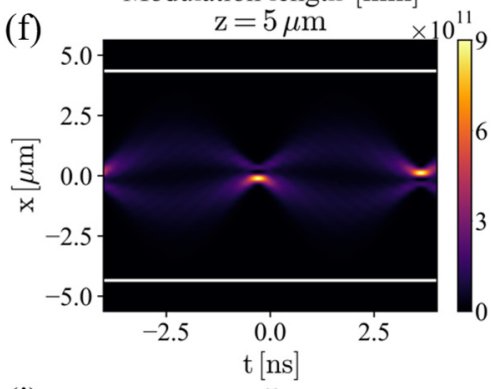

(i)

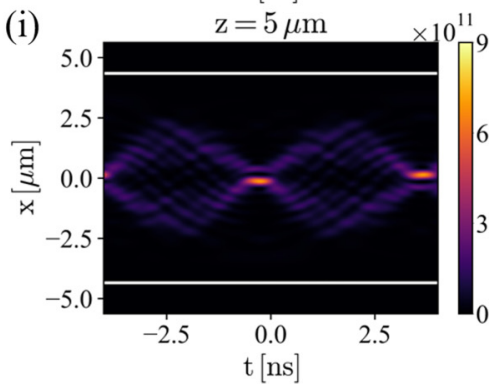

FIG. 3. Generate slow guided space-time wave packets with $v_{g}=1 \times 10^{-5} \mathrm{c}$. (a) The initial state $(v=2$, red cross) and the selected eigenmodes (green dots) on the dispersion relation (blue curves). $\omega_{b} / c=4.2 \mu \mathrm{m}^{-1}$. (b) The intensity profile of the initial state. (c) The evolution of the mode amplitudes $(v=2,5,20)$ under modulation. (d)-(f) The target state: (d) the mode amplitudes; (e), (f) the intensity profile at $z=0$ and $z=5 \mu \mathrm{m}$. (g)-(i) The corresponding results for the generated state.

We consider the $v=2$ eigenmode to be the initial state [red cross in Fig. 3(a)], as it leads to the highest maximum fidelity $F=0.9$ determined by Eq. (15) among all the eigenmodes. Figure 3(b) shows the intensity distribution of the initial state. We send the initial state into a modulation region of length $L_{m}=59.70 \mathrm{~mm}$. In the modulation region, the state undergoes complicated evolution [Fig. 3(c)]. At the end of the modulation region, the mode amplitudes of the transmitted state [Fig. $3(\mathrm{~g})]$ are similar to those of the target state [Fig. 3(d)]. The obtained fidelity for the transmitted state is $F(z=0)=0.861$, which is very close to the maximum fidelity as quoted above. Here $z=0$ denotes the position immediately after the modulation region. Upon exiting the modulation region, the pulse propagates further in an unmodulated waveguide. Figures 3(h) and 3(i) show the intensity distribution of the final state at $z=0$ and $z=5 \mu \mathrm{m}$, respectively, which are similar to Figs. 3(e) and 3(f). These plots clearly show that we have generated propagation-invariant guided space-time wave packets with desired group velocity $v_{g}^{(1)}=1 \times 10^{-5} \mathrm{c}$ and field profile.

In the second example, we use the modulation of the case (2) to generate a train of "backward" propagating space-time wave packets with a group velocity $v_{g}^{(2)}=-2 \times 10^{-5} \mathrm{c}$. We note that the negative group velocity refers to the velocity of (a)

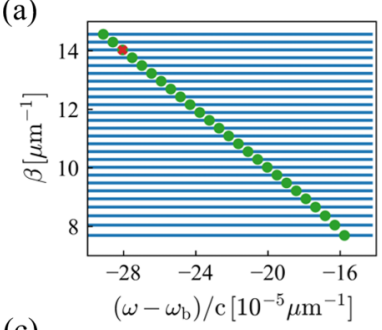

(c)

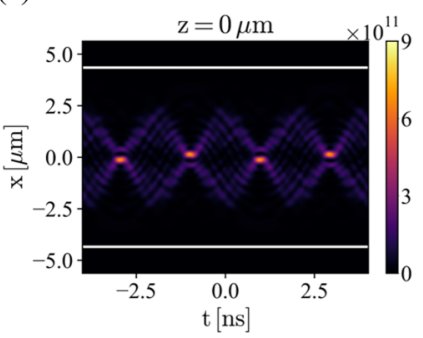

(b)

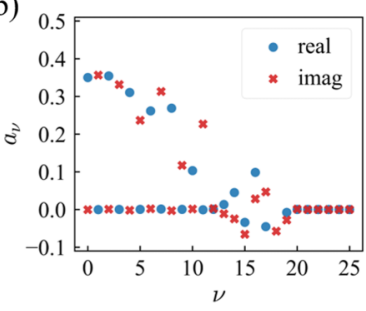

(d)

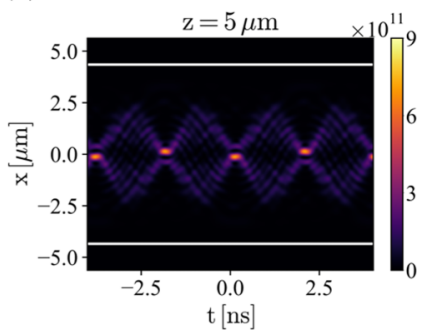

FIG. 4. Generate backward-guided space-time wave packets with $v_{g}=-2 \times 10^{-5} \mathrm{c}$. (a) The initial state $(v=2$, red cross) and selected eigenmodes (green dots) on the band diagram (blue curves). $\omega_{b} / c=4.2 \mu \mathrm{m}^{-1}$. (b) The mode amplitudes of the generated state. (c), (d) The intensity profile of the generated state at $z=0$ and $z=5 \mu \mathrm{m}$. 
(a)

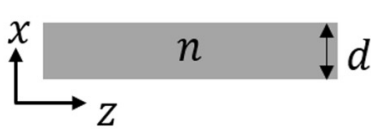

(d)

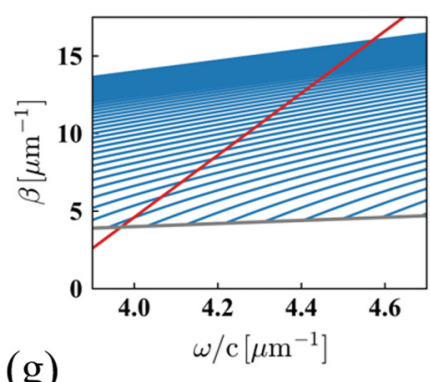

$(\mathrm{g})$

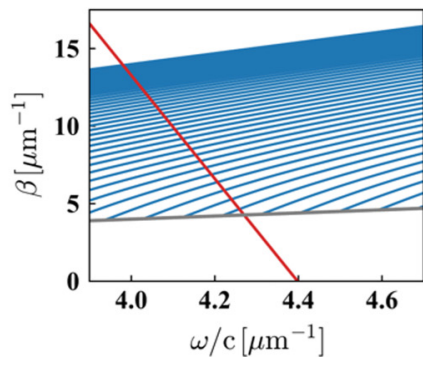

(b)

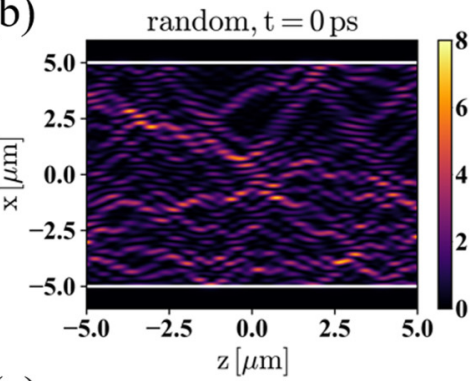

(e)

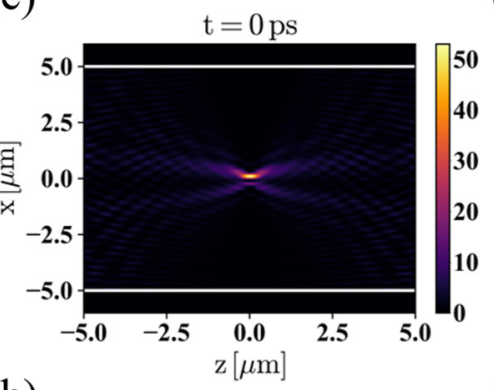

(h)

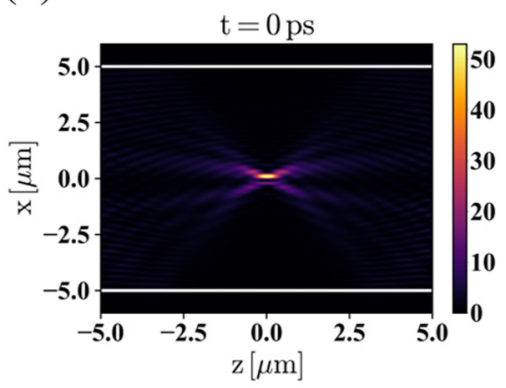

(c)

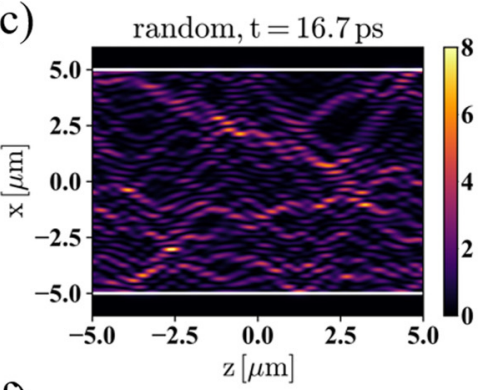

(f)

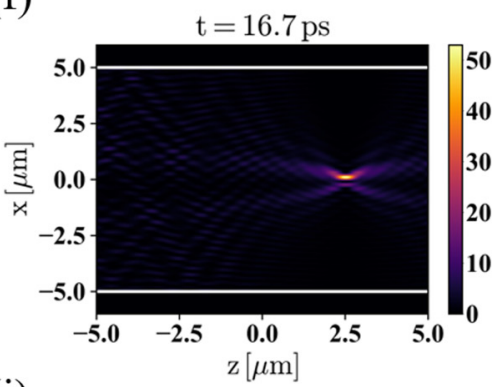

(i)

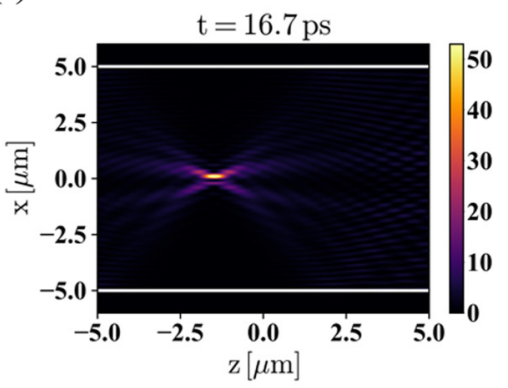

FIG. 5. Guided space-time wave packets in a slab waveguide. (a) Structure. The refractive index $n=3.5$ and thickness $d=10 \mu \mathrm{m}$. (b), (c) The intensity profile $|E(x, z, t)|^{2}$ for light with random mode amplitudes $\left\{a_{v}\right\}$ at $t=0 \mathrm{ps}$ and $t=16.7 \mathrm{ps}$. The selected modes are given in (d). (d)-(f) A slow guided space-time wave packet. (d) Blue curves: band diagram of the static waveguide. Red straight line: the spatiotemporal correlation $\omega=v_{g} \beta+\omega_{b}$, where $v_{g}=0.05 \mathrm{c}, \omega_{b} / c=3.77 \mu \mathrm{m}^{-1}$. A set of eigenstates are selected at the intersections. (e), (f) The intensity profile at $t=0 \mathrm{ps}$ and $t=16.7 \mathrm{ps}$. The mode amplitudes $a_{v}=1$ for all the selected eigenmodes. (g)-(i) A backward-guided space-time wave packet. Similar to (d)-(f) except that $v_{g}=-0.03 \mathrm{c}, \omega_{b} / c=4.40 \mu \mathrm{m}^{-1}$.

intensity peaks for each individual wave packet. The Poynting vector is still along the $+z$ direction $[40,60]$.

The modulation induces photonic transitions among a set of eigenmodes [green dots in Fig. 4(a)] that approximately satisfy the spatiotemporal correlation

$$
\omega_{v}^{(2)}-\omega_{0}^{(2)}=v_{p}^{(2)}\left(\beta_{v}^{(2)}-\beta_{0}^{(2)}\right),
$$

where $\quad \omega_{0}^{(2)} / c=4.2-30.11 \times 10^{-5} \mu \mathrm{m}^{-1}, \quad \beta_{0}^{(2)}=$ $14.5659 \mu \mathrm{m}^{-1}$, and $v_{g}^{(2)}=v_{p}^{(2)}=-2 \times 10^{-5} \mathrm{c}$. We set a target state with the same $\left\{a_{v}\right\}$ as Eq. (28) and Fig. 3(d) for the first case. Although $\left\{a_{v}\right\}$ is the same for the two cases, due to the different phase velocity of the traveling wave modulation, the selected eigenmodes have different frequencies and wave vectors, the group velocities of the optical pulses are drastically different.

We again consider the $v=2$ eigenmode as the initial state. We send it into a modulation region of length $L_{m}=59.70 \mathrm{~mm}$. The state undergoes similar evolution as the case (1), because the selected eigenmode profiles, and thus the Hamiltonian, are very similar in the two cases. The transmitted state has a mode amplitude distribution [Fig. 4(b)] very close to Fig. 3(g). Fig- ures 4(e) and 4(f) show the intensity distribution of the final state at $z=0$ and $z=5 \mu \mathrm{m}$, respectively. The intensity peaks indeed propagate backward by $\tau^{(2)}=\Delta z /\left|v_{g}^{(2)}\right|=0.83 \mathrm{~ns}$.

\section{DISCUSSION AND CONCLUSION}

In this work, we have primarily focused on monochromatic modulation in a specific GRIN waveguide to highlight the physics. Our method can be readily extended to polychromatic modulation in waveguides with other refractive index profiles. In such systems, a single pulsed space-time wave packet can be generated. (See the Appendix for more discussion.)

In conclusion, we have proposed an integrated photonic approach to generating guided space-time wave packets in a conventional multimode waveguide based on multilevel interband photonic transitions. It can generate broadband propagation-invariant space-time wave packets with arbitrary group velocity and tailored field profiles. Our work reveals a connection between the study of time-varying media and space-time optics, and may enable integrated photonic applications of guided space-time wave packets. 


\section{ACKNOWLEDGMENTS}

This work is supported by a MURI project from the U.S. Air Force Office of Scientific Research (FA9550-18-1-0379).

\section{APPENDIX}

In the main text, we have primarily focused on a specific GRIN waveguide to highlight the physics. Using such a GRIN waveguide, we demonstrate a train of guided space-time wave packets. Our method can be readily extended to waveguides with other refractive index profiles. In such systems, a single pulsed space-time wave packet can be generated. Here we provide a concrete example of such systems. We also use this example to highlight the importance of the complex amplitude distribution on the field profile.

We consider a simple slab waveguide with a refractive index $n=3.5$ and width $d=10 \mu \mathrm{m}$ [Fig. 5(a)]. This waveguide supports many modes in the telecom frequency range [blue curves in Figs. 5(d) and 5(g)]. Importantly, the propagation constants are not equally spaced. The spatiotemporal correlation $\omega=v_{g} \beta+\omega_{b}$ is represented by a straight line in the band diagram. The intersections of the straight line and band dispersions select a set of eigenstates, one for each band. First, we consider slow light where $v_{g}=0.05 \mathrm{c}$, $\omega_{b} / c=3.77 \mu \mathrm{m}^{-1}$, and the complex amplitude $a_{\nu}=1$ for all the 43 selected eigenmodes [Figs. 5(d)-5(f)]. Figures 5(e) and 5 (f) show the intensity profile $|E(x, z, t)|^{2}$ at $t=0 \mathrm{ps}$ and $t=16.7 \mathrm{ps}$, respectively. The wave packet is a single pulse strongly localized near the waveguide center with a full width at half maximum $\Delta z=0.50 \mu \mathrm{m}$ and $\Delta x=0.20 \mu \mathrm{m}$. The wave packet is a single pulse rather than a pulse train because the propagation constants are not equally spaced. The short pulse width is due to the broad bandwidth determined by the frequency span of the selected eigenmodes (190-220 THz). Indeed, the wave packet propagates without distortion at a group velocity $v_{g}=0.05 \mathrm{c}$, as inferred from Figs. 5(e) and 5(f). Second, we consider fast (backward) light where $v_{g}=-0.03 \mathrm{c}, \omega_{b} / c=4.40 \mu \mathrm{m}^{-1}$, and $a_{v}=1$ for all the 46 selected eigenmodes [Figs. 5(g)-5(i)]. Figures 5(h) and 5(i) plot $|E(x, z, t)|^{2}$ at $t=0 \mathrm{ps}$ and $t=16.7 \mathrm{ps}$, respectively. The wave packet propagates without distortion at a group velocity $v_{g}=-0.03 \mathrm{c}$.

Finally, we highlight how the mode amplitudes govern the field profile. We consider light with the same spatiotemporal correlation as shown in Fig. 5(d), but now with a random distribution of $\left\{a_{v}\right\}$. Figures $5(\mathrm{~b})$ and 5(c) plot $|E(x, z, t)|^{2}$ at $t=0 \mathrm{ps}$ and $t=16.7 \mathrm{ps}$, respectively. The intensity distributions are random, which are completely different from Figs. 5(e) and 5(f). Nonetheless, the whole intensity pattern still propagates rigidly at a group velocity $v_{g}=0.05 \mathrm{c}$.
[1] M. F. Yanik and S. Fan, Stopping Light all Optically, Phys. Rev. Lett. 92, 083901 (2004).

[2] M. F. Yanik and S. Fan, Time Reversal of Light with Linear Optics and Modulators, Phys. Rev. Lett. 93, 173903 (2004).

[3] Y. Xiao, D. N. Maywar, and G. P. Agrawal, Reflection and transmission of electromagnetic waves at a temporal boundary, Opt. Lett. 39, 574 (2014).

[4] Y. Hadad, D. L. Sounas, and A. Alu, Space-time gradient metasurfaces, Phys. Rev. B 92, 100304(R) (2015).

[5] J. S. Martínez-Romero, O. M. Becerra-Fuentes, and P. Halevi, Temporal photonic crystals with modulations of both permittivity and permeability, Phys. Rev. A 93, 063813 (2016).

[6] A. M. Shaltout, V. M. Shalaev, and M. L. Brongersma, Spatiotemporal light control with active metasurfaces, Science $\mathbf{3 6 4}$, eaat3100 (2019).

[7] P. A. Huidobro, E. Galiffi, S. Guenneau, R. V. Craster, and J. B. Pendry, Fresnel drag in space-time-modulated metamaterials, Proc. Natl. Acad. Sci. USA 116, 24943 (2019).

[8] N. Engheta, Metamaterials with high degrees of freedom: Space, time, and more, Nanophotonics 10, 639 (2021).

[9] C. Caloz and Z.-L. Deck-Léger, Spacetime metamaterialsPart II: Theory and applications, IEEE Trans. Antennas Propag. 68, 1583 (2020).

[10] T. T. Koutserimpas and R. Fleury, Electromagnetic fields in a time-varying medium: Exceptional points and operator symmetries, IEEE Trans. Antennas Propag. 68, 6717 (2020).

[11] J. N. Winn, S. Fan, J. D. Joannopoulos, and E. P. Ippen, Interband transitions in photonic crystals, Phys. Rev. B 59, 1551 (1999).

[12] P. Dong, S. F. Preble, J. T. Robinson, S. Manipatruni, and M. Lipson, Inducing Photonic Transitions between Discrete Modes in a Silicon Optical Microcavity, Phys. Rev. Lett. 100, 033904 (2008).

[13] Z. Yu and S. Fan, Complete optical isolation created by indirect interband photonic transitions, Nat. Photonics 3, 91 (2009).

[14] M. Castellanos Muñoz, A. Y. Petrov, L. O’Faolain, J. Li, T. F. Krauss, and M. Eich, Optically Induced Indirect Photonic Transitions in a Slow Light Photonic Crystal Waveguide, Phys. Rev. Lett. 112, 053904 (2014).

[15] E. A. Kittlaus, N. T. Otterstrom, P. Kharel, S. Gertler, and P. T. Rakich, Non-reciprocal interband Brillouin modulation, Nat. Photonics 12, 613 (2018).

[16] D. L. Sounas and A. Alù, Non-reciprocal photonics based on time modulation, Nat. Photonics 11, 774 (2017).

[17] I. A. D. Williamson, M. Minkov, A. Dutt, J. Wang, A. Y. Song, and S. Fan, Integrated nonreciprocal photonic devices with dynamic modulation, Proc. IEEE 108, 1759 (2020).

[18] M. S. Kang, A. Butsch, and P. S. J. Russell, Reconfigurable light-driven opto-acoustic isolators in photonic crystal fibre, Nat. Photonics 5, 549 (2011).

[19] H. Lira, Z. Yu, S. Fan, and M. Lipson, Electrically Driven Nonreciprocity Induced by Interband Photonic Transition on a Silicon Chip, Phys. Rev. Lett. 109, 033901 (2012).

[20] D. L. Sounas, C. Caloz, and A. Alù, Giant non-reciprocity at the subwavelength scale using angular momentum-biased metamaterials, Nat. Commun. 4, 2407 (2013).

[21] N. A. Estep, D. L. Sounas, J. Soric, and A. Alù, Magnetic-free non-reciprocity and isolation based on parametrically modulated coupled-resonator loops, Nat. Phys. 10, 923 (2014).

[22] Y. Shi, Q. Lin, M. Minkov, and S. Fan, Nonreciprocal optical dissipation based on direction-dependent Rabi splitting, IEEE J. Sel. Top. Quantum Electron. 24, 1 (2018). 
[23] Y. Shi, S. Han, and S. Fan, Optical circulation and isolation based on indirect photonic transitions of guided resonance modes, ACS Photonics 4, 1639 (2017).

[24] K. Fang, Z. Yu, and S. Fan, Realizing effective magnetic field for photons by controlling the phase of dynamic modulation, Nat. Photonics 6, 782 (2012).

[25] L. D. Tzuang, K. Fang, P. Nussenzveig, S. Fan, and M. Lipson, Non-reciprocal phase shift induced by an effective magnetic flux for light, Nat. Photonics 8, 701 (2014).

[26] K. Fang, Z. Yu, and S. Fan, Photonic Aharonov-Bohm Effect Based on Dynamic Modulation, Phys. Rev. Lett. 108, 153901 (2012).

[27] K. Fang, Z. Yu, and S. Fan, Experimental demonstration of a photonic Aharonov-Bohm effect at radio frequencies, Phys. Rev. B 87, 060301(R) (2013).

[28] E. Li, B. J. Eggleton, K. Fang, and S. Fan, Photonic AharonovBohm effect in photon-phonon interactions, Nat. Commun. 5, 3225 (2014).

[29] H. E. Kondakci and A. F. Abouraddy, Diffraction-free spacetime light sheets, Nat. Photonics 11, 733 (2017).

[30] M. Yessenov, B. Bhaduri, H. E. Kondakci, and A. F. Abouraddy, Weaving the rainbow: Space-time optical wave packets, Opt. Photonics News 30, 34 (2019).

[31] M. Yessenov, B. Bhaduri, H. E. Kondakci, and A. F. Abouraddy, Classification of propagation-invariant space-time wave packets in free space: Theory and experiments, Phys. Rev. A 99, 023856 (2019).

[32] Y. Silberberg, Collapse of optical pulses, Opt. Lett. 15, 1282 (1990).

[33] J.-Y. Lu and J. Greenleaf, Nondiffracting X waves-exact solutions to free-space scalar wave equation and their finite aperture realizations, IEEE Trans. Ultrason. Ferroelectr. Freq. Control 39, 19 (1992).

[34] P. Saari and K. Reivelt, Evidence of x-Shaped PropagationInvariant Localized Light Waves, Phys. Rev. Lett. 79, 4135 (1997).

[35] H. E. Hernández-Figueroa, M. Zamboni-Rached, and E. Recami (eds.), Localized Waves (Wiley-Interscience, IEEE Press, Hoboken, NJ, 2008).

[36] J. Turunen and A. T. Friberg, Propagation-invariant optical fields, in Progress in Optics, Vol. 54 (Elsevier, Oxford, 2010), pp. 1-88.

[37] D. Abdollahpour, S. Suntsov, D. G. Papazoglou, and S. Tzortzakis, Spatiotemporal Airy Light Bullets in the Linear and Nonlinear Regimes, Phys. Rev. Lett. 105, 253901 (2010).

[38] A. Chong, W. H. Renninger, D. N. Christodoulides, and F. W. Wise, Airy-Bessel wave packets as versatile linear light bullets, Nat. Photonics 4, 103 (2010).

[39] H. E. Hernández-Figueroa, E. Recami, and M. ZamboniRached (eds.), Non-Diffracting Waves (Wiley-VCH Verlag, Weinheim, Germany, 2014).

[40] H. E. Kondakci and A. F. Abouraddy, Optical space-time wave packets having arbitrary group velocities in free space, Nat. Commun. 10, 929 (2019).

[41] C. Guo, M. Xiao, M. Orenstein, and S. Fan, Structured 3D linear space-time light bullets by nonlocal nanophotonics, Light Sci Appl 10, 160 (2021).
[42] B. Bhaduri, M. Yessenov, and A. F. Abouraddy, Space-time wave packets that travel in optical materials at the speed of light in vacuum, Optica 6, 139 (2019).

[43] B. Bhaduri, M. Yessenov, and A. F. Abouraddy, Anomalous refraction of optical spacetime wave packets, Nat. Photonics 14, 416 (2020).

[44] A. Shiri, M. Yessenov, S. Webster, K. L. Schepler, and A. F. Abouraddy, Hybrid guided space-time optical modes in unpatterned films, Nat. Commun. 11, 6273 (2020).

[45] K. L. Schepler, M. Yessenov, Y. Zhiyenbayev, and A. F Abouraddy, Space-time surface plasmon polaritons: A new propagation-invariant surface wave packet, ACS Photonics 7, 2966 (2020).

[46] B. Kibler and P. Béjot, Discretized Conical Waves in Multimode Optical Fibers, Phys. Rev. Lett. 126, 023902 (2021).

[47] M. Yessenov, B. Bhaduri, H. E. Kondakci, M. Meem, R. Menon, and A. F. Abouraddy, Non-diffracting broadband incoherent space-time fields, Optica 6, 598 (2019).

[48] H. Weyl, Über die Gleichverteilung von Zahlen mod. Eins, Math. Ann. 77, 313 (1916).

[49] A. M. Samoilenko, Mathematics and its Applications, Elements of the Mathematical Theory of Multi-Frequency Oscillations, Mathematics and its Applications, Vol. 71 (Springer Netherlands, Dordrecht, 1991).

[50] T. Tamir (ed.), Topics in Applied Physics, Integrated Optics, Topics in Applied Physics, Vol. 7 (Springer, Berlin, 1975).

[51] M. J. Adams, An Introduction to Optical Waveguides (Wiley, Chichester, 1981).

[52] A. W. Snyder and J. D. Love, Optical Waveguide Theory (Chapman and Hall, London, 1983).

[53] K. Okamoto, Fundamentals of Optical Waveguides, 2nd ed. (Elsevier, Amsterdam, 2006).

[54] C. Gomez-reino, M. V. Perez, C. Bao, M. T. Flores-arias, and S. Vidal, Diffraction-free and diffraction-limited propagation of light in graded-index planar waveguides with hyperbolic secant refractive index profile, J. Mod. Opt. 47, 91 (2000).

[55] X. Wang, C. Yin, and Z. Cao, Progress in Planar Optical Waveguides (Jiao Tong University Press, Shanghai, 2016).

[56] C. Wang, M. Zhang, X. Chen, M. Bertrand, A. ShamsAnsari, S. Chandrasekhar, P. Winzer, and M. Lončar, Integrated lithium niobate electro-optic modulators operating at CMOS-compatible voltages, Nature (London) 562, 101 (2018).

[57] Q. Lin, J. Wang, and S. Fan, Compact dynamic optical isolator based on tandem phase modulators, Opt. Lett. 44, 2240 (2019).

[58] M. Zhang, C. Wang, P. Kharel, D. Zhu, and M. Lončar, Integrated lithium niobate electro-optic modulators: When performance meets scalability, Optica 8, 652 (2021).

[59] A. Mohanty, M. Zhang, A. Dutt, S. Ramelow, P. Nussenzveig, and M. Lipson, Quantum interference between transverse spatial waveguide modes, Nat. Commun. 8, 14010 (2017).

[60] M. Yessenov, L. Mach, B. Bhaduri, D. Mardani, H. E. Kondakci, G. K. Atia, M. A. Alonso, and A. F. Abouraddy, What is the maximum differential group delay achievable by a space-time wave packet in free space? Opt. Express 27, 12443 (2019). 